\title{
Severe cardiomyopathy induced by Adalimumab administration for Crohn's disease
}

\author{
Ali Toufaily ${ }^{1,2 *}$ \\ ${ }^{1}$ Division of Cardiology, Faculty of Medical Sciences, Lebanese University, Hadath, Lebanon \\ ${ }^{2}$ Division of Cardiology, Rafik Hariri University Hospital, Beirut, Lebanon
}

\begin{abstract}
The use of tumor necrosis factor alpha inhibitors anti-TNFa (infliximab, adalimumab, and certolizumab) in the treatment of inflammatory bowel disease (IBD) provide a major therapeutic advance, allowing significant benefits in the induction and maintenance of remission in Crohn's disease [1-3]. However, the safety-profile of TNF inhibitors with regard to worsening or new onset of heart failure is still very controversial. Consequently, there has been a lot of attention paid to the risk of cardiovascular side effects associated with adalimumab therapy [4-6]. A 42-year-old woman treated with adalimumab for severe Crohn's disease exacerbation; 8 days after the second dose of adalimumab $(80 \mathrm{mg})$, she presented with dramatic clinical features of heart failure and pulmonary edema. Echocardiography demonstrated severely depressed left ventricle contractility with ejection fraction 35\%; cardiac MRI revealed no gadolinium enhancement (no ischemia, no myocarditis). Adalimumab was discontinued, and patient was treated with evidence-based oral disease-modifying HF therapy; 2 months later, cardiac function has returned to normal. This case clearly demonstrated the acute onset of decompensated heart failure with adalimumab usage.
\end{abstract}

\section{Introduction}

Inhibitors of tumor necrosis factor (TNF)-alpha (adalimumab) offer an important targeted therapy in a large number of inflammatory conditions, including rheumatoid arthritis (RA), psoriasis, spondyloarthritis and inflammatory bowel disease (IBD) [7,8]. In fact, the effectiveness of those drugs has been well established. However, multiple adverse effects have been identified through both clinical trials and post-marketing surveillance, ranging from benign to serious side effect such as lymphomas, infections (especially reactivation of latent tuberculosis), demyelinating disease and cardiomyopathy which are associated with substantial mortality $[9,10]$.

\section{Case Report}

A 42-year-old woman, former smoker, non-alcoholic has been diagnosed with Crohn's disease since teenage, maintained on azathioprine. Apart from her gastro-intestinal symptoms, her prior medical history was unremarkable. She was admitted to our ward for profuse watery diarrhea with abdominal pain, managed as acute exacerbation of her Crohn's disease. At that time adalimumab $160 \mathrm{mg}$ was initiated and she had improved a lot from her baseline. Two weeks later, she received a dose of $80 \mathrm{mg}$ subcutaneous injection according to the universal treatment regimen of adalimumab, then after 8 days, she presented to our ER department with severe shortness of breath, palpitation, cough, and desaturation. Echocardiographic examination showed severe left ventricle function impairment with ejection fraction $35 \%$, moderate mitral regurgitation with mild dilatation of LV cavity. The laboratory findings showed elevated troponin, high pro BNP level, mild anemia, normal WBC count, normal TSH and D dimer levels.

Coronary angiography was made showing non stenotic coronaries (Figure 1). Cardiac MRI confirmed the impaired global contractility of the LV, with no late gadolinium enhancement (Figure 2).Taking into consideration the last echocardiography performed 1 month ago which

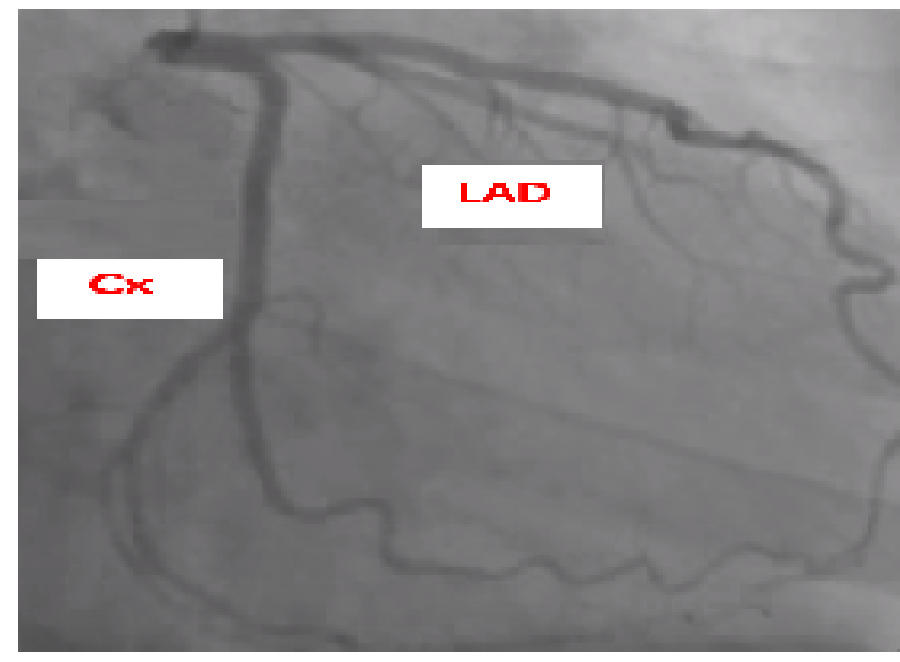

Figure 1. Normal angiogram

was totally normal in addition to non-specific MRI features obtained, as an exclusion diagnosis, adalimumab was considered as the cause of the acute decompensated heart failure. Patient was placed on optimal heart failure therapy then discharged after appropriate stabilization.

Adalimumab remained certainly withdrawn then 2 months later, repeated echocardiography demonstrated a full recovery.

${ }^{\star}$ Correspondence to: Ali Toufaily, Division of Cardiology, Faculty of Medical Sciences, Lebanese University, Lebanon, E-mail: alitfayli81@gmail.com

Key words: TNF-a inhibitor, adalimumab, heart failure, crohn's disease

Received: December 16, 2019; Accepted: December 26, 2019; Published: January 03, 2020 


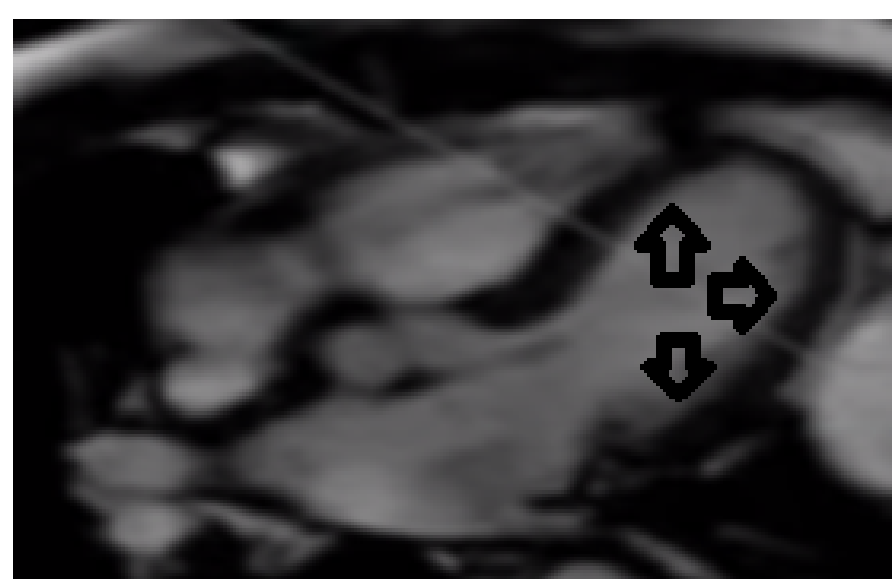

Figure 2. No LGE on cardiac MRI

\section{Discussion and Conclusion}

Adalimumab have dramatically improved the therapy of many inflammatory diseases and led to major progress not only for patients with Crohn's disease but also for those with ulcerative colitis, psoriasis, ankylosing spondylitis and rheumatoid arthritis [11-13]. Common side effects such as headache, rash and nausea are usually well tolerated and self-limiting and generally do not lead to drug discontinuation. However, disabling and potentially life-threatening side effects occurred as well, particularly opportunistic infection, tumor development, and cardiotoxicity. Although adalimumab induced tachyarrhythmias, hypertension or palpitation were frequently reported, the link between TNF blockade and moderate to severe congestive heart failure remains controversial $[14,15]$. In daily practice, the physicians must at all times be alert to the possible occurrence of adalimumab induced cardiomyopathy after careful exclusion of others etiologies such as myocarditis, myocardial infarction, stress induced cardiomyopathy. In conclusion, further research seems to be warranted to well define the cardiotoxicity profile of adalimumab.

\section{References}

1. D'Haens GR, Panaccione R, Higgins PD, Vermeire S, Gassull M, et al. (2011) The London Position Statement of the World Congress of Gastroenterology on Biological Therapy for IBD with the European Crohn's and Colitis Organization: when to start, when to stop, which drug to choose, and how to predict response? Am J Gastroenterol 106: 199 -212. [Crossref]
2. Lichtenstein GR, Loftus EV, Isaacs KL, Regueiro MD, Gerson LB, et al. (2018) ACG Clinical Guideline: Management of Crohn's Disease in Adults. Am J Gastroenterol 113: 481- 517. [Crossref]

3. Terdiman JP, Gruss CB, Heidelbaugh JJ, Sultan S, Falck-Ytter YT, et al. (2013) American Gastroenterological Association Institute guideline on the use of thiopurines, methotrexate, and anti-TNF- $\alpha$ biologic drugs for the induction and maintenance of remission in inflammatory Crohn's disease. Gastroenterology 145: 1459-1463. [Crossref]

4. Tracey D, Klareskog L, Sasso E.H, Salfeld J.G, Tak P.P. (2008) Tumor necrosis factor antagonist mechanisms of action: A comprehensive review. Pharmacol. Ther 117: 244-279. [Crossref]

5. Colombel JF, Sandborn WJ, Rutgeerts P, Enns R, Hanauer SB, et al. (2007) Adalimumab for maintenance of clinical response and remission in patients with Crohn's disease: The CHARM trial. Gastroenterology 132: 52-65. [Crossref]

6. Osterman MT, Haynes K, Delzell E, Zhang J, Bewtra M, et al. (2014) Comparative Effectiveness of Infliximab and Adalimumab for Crohn's Disease Critical revision of the manuscript for important intellectual content Statistical analysis Obtained funding. Clin. Gastroenterol Hepatol 12: 811-817. [Crossref]

7. Kuek A, Hazleman BL, Ostor AJ (2007) Immune-mediated inflammatory diseases (IMIDs) and biologic therapy: A medical revolution. Postgrad Med J 83: 251-260. [Crossref]

8. Silva LC, Ortigosa LC, Benard G (2010) Anti-TNF-alpha agents in the treatment of immune-mediated inflammatory diseases: Mechanisms of action and pitfalls. Immunotherapy 2: 817-833. [Crossref]

9. Siegel CA (2010) The risks of biologic therapy for inflammatory bowel disease. In: Bernstein ED, editor. The Inflammatory Bowel Disease Yearbook, volume 6. London, UK: Remedica: 89-108.

10. Ferkolj I. (2009) How to improve the safety of biologic therapy in Crohn's disease. $J$ Physiol Pharmacol 60: 67-70. [Crossref]

11. Hanauer SB, D'Haens GR, Colombel JF, Sandborn WJ, Rutgeerts P, et al. (2006a) Sustained clinical remission in patients with moderate to severe Crohn's disease with adalimumab, regardless of anti-TNF history or concomitant immunosuppressant therapy. Am J Gastroenterol 101: pS457.

12. Chen YF, Jobanputra P, Barton P, Jowett $S$, Bryan S, et al. (2006) A systematic review of the effectiveness of adalimumab, etanercept and infliximab for the treatment of rheumatoid arthritis in adults and an economic evaluation of their cost-effectiveness. Health Technol Assess 10: 1-229. [Crossref]

13. Gordon KB, Langley RG, Leonardi C, Toth D, Menter MA, et al. (2006) Clinica response to adalimumab treatment in patients with moderate to severe psoriasis: double-blind, randomized controlled trial and open-label extension study. $J$ Am Acad Dermatol 55: 598-606. [Crossref]

14. Kwon HJ, Cote TR, Cuffe MS, Kramer JM, Braun MM, et al. (2003) Case reports of heart failure after therapy with a tumor necrosis factor antagonist. Ann Intern Med 138: 807-811. [Crossref]

15. Cacciapaglia F, Navarini L, Menna P, Salvatorelli E, Minotti G, et al. (2011) Cardiovascular safety of anti-TNF-alpha therapies: facts and unsettled issues. Autoimmune Rev 10: 631-635. [Crossref]

Copyright: (C2020 Toufaily A. This is an open-access article distributed under the terms of the Creative Commons Attribution License, which permits unrestricted use, distribution, and reproduction in any medium, provided the original author and source are credited. 